\title{
Muscle transcriptome adaptations with mild eccentric ergometer exercise
}

\author{
Stephan Klossner • Christoph Däpp • Silvia Schmutz • \\ Michael Vogt • Hans Hoppeler • Martin Flück
}

Received: 24 January 2007 / Revised: 12 April 2007 / Accepted: 29 May 2007 / Published online: 16 August 2007

(C) Springer-Verlag 2007

\begin{abstract}
The muscle has a wide range of possibilities to adapt its phenotype. Repetitive submaximal concentric exercise (i.e., shortening contractions) mainly leads to adaptations of muscle oxidative metabolism and endurance while eccentric exercise (i.e., lengthening contractions) results in muscle growth and gain of muscle strength. Modified gene expression is believed to mediate these exercise-specific muscle adjustments. In the present study, early alterations of the gene expression signature were monitored by a muscle-specific microarray. Transcript profiling was performed on muscle biopsies of vastus lateralis obtained from six male subjects before and in a 24-h time course after a single bout of mild eccentric ergometer exercise. The eccentric exercise consisted of $15 \mathrm{~min}$ of eccentric cycling at $50 \%$ of the individual maximal concentric power output leading to muscle soreness (5.9 on a $0-10$ visual analogue scale) and limited muscle damage (1.7-fold elevated creatine kinase activity). Muscle impairment was highlighted by a transient reduction in jumping height after the eccentric exercise. On the gene expression level, we observed a general early downregulation of detected transcripts, followed by a slow recovery close to the control values within the first $24 \mathrm{~h}$ post
\end{abstract}

Electronic supplementary material The online version of this article (doi:10.1007/s00424-007-0303-6) contains supplementary material, which is available to authorized users.

S. Klossner $(\bowtie) \cdot$ C. Däpp · S. Schmutz $\cdot$ M. Vogt $\cdot$ H. Hoppeler $\cdot$ M. Flück

Institute of Anatomy, University of Berne,

Berne, Switzerland

e-mail: klossner@ana.unibe.ch

\section{Flück}

Institute for Biophysical and Clinical Research into Human

Movement, Manchester Metropolitan University,

Manchester, UK exercise. Only very few regulatory factors were increased. This expression signature is different from the signature of a previously published metabolic response after an intensive endurance-type concentric exercise as well as after maximal eccentric exercise. This is the first description of the time course of changes in gene expression as a consequence of a mild eccentric stimulus.

Keywords Human - Skeletal muscle - Eccentric exercise . Gene expression $\cdot$ Microarray $\cdot$ Muscle damage

\section{Introduction}

Exercise induces phenotypical adaptations in skeletal muscle, which critically depends on specific mechanical, metabolic, and hormonal responses elicited by the stimulus. This is illustrated by a different response of mitochondria and contractile elements in muscles after long-term lowload high-repetitive "endurance-type" exercise [13] vs highload low-repetitive "strength-type" exercise [28, 44].

We have shown that endurance training causes improvements in oxidative metabolic characteristics including enhanced capillary and myocellular lipid metabolism as well as changes in glycogen metabolism [37]. Recent research demonstrates that the mechanisms responsible for the adaptive processes are reflected by modifications in gene expression. In particular, it was shown that ribonucleic acid (RNA) concentrations of mitochondrial transcripts are increased approximately in proportion to the gain in mitochondrial volume [33]. The idea of a dominant control of muscle phenotype through an increase in muscle gene expression was supported by the group of Pilegaard [31], who performed nuclear run-on assays after concentric endurance type exercise. These experiments furnished 
direct evidence of the transcriptional control of RNA concentrations in training studies. Further evidence came from Hood et al. [18], who elucidated the mechanisms by which mitochondrial biogenesis is regulated and integrated in an exercise setting [31]. Looking at the time course of the transcript response to a single concentric exercise bout, Schmutz et al. [37] could demonstrate that a majority of muscle transcripts were upregulated after $8 \mathrm{~h}$ of recovery, with most transcript levels returning to baseline values after $24 \mathrm{~h}$ [37].

In strength training, the dominant phenotypical adaptation of the skeletal muscle tissue consists of an increase in myofibrils and associated proteins. This is classically achieved by high-load muscle contractions [36]. More recently, it was shown that considerable gains in muscle strength and fiber cross-sectional area can also be achieved by chronic eccentric exercise (medium-load high-repetitive negative work $=$ mild eccentric ergometer exercise) $[17,20$, 25]. This finding is of great practical implication, as chronic eccentric exercise could be used as an important mean to increase muscle performance and muscle mass in situations where high-load exercise may not be tolerated [27, 38]. The mild eccentric exercise analyzed in this study circumvents muscle damage and pain while maintaining the mechanical stress of the eccentric muscle contractions and its potential gain in muscle strength. Currently, there are few data on the molecular mechanisms underlying the phenotypical adaptations to eccentric exercise. Studies using low-repetitive high-load eccentric exercise indicate that gene transcripts involved in extracellular remodeling and the inflammatory response are strongly increased up to $24 \mathrm{~h}$ postexercise [7]. The same group showed a direct relation between the mechanical load and the activation of the inflammatory response [8]. These results indicate that high mechanical stress experienced by muscles during heavy eccentric exercise leads to a specific molecular response, different to that after concentric exercise but similar in time course.

It was our aim to characterize the specific changes of the muscle transcriptome to a single mild eccentric stimulus, which was previously shown to increase muscle cross-sectional area and muscle strength, when applied repetitively over longer time periods [25]. We hypothesized that eccentric exercise would result in a broad upregulation of transcripts relevant for the processes initiating muscle growth and improved strength generation within the first $24 \mathrm{~h}$ postexercise.

\section{Materials and methods}

Subjects and training intervention

This study was conducted with permission of the Ethics Committee of Bern, Switzerland, in compliance with the Helsinki Convention for Research on human subjects. Six untrained male subjects gave their written consent to participate in the study. They were recruited to perform a single bout of chronic negative work (eccentric exercise) on a custom-built eccentric-bike (e-bike [27, 38]). The e-bike is driven by a 5 -hp motor that regulates pedal revolutions per minute and torque. The subject had to perform eccentric muscle contractions by resisting the motor, such that the applied eccentric torque developed by the leg was equal to a given target load displayed on a computer monitor. Anthropometric parameters such as age, height, weight, lean body mass, and body mass index (BMI) were determined at the outset of the study. The subjects performed a $\mathrm{VO}_{2 \max }$ test estimating their maximal concentric power output $\left(P_{\max }\right)$. Two weeks later, they performed a single eccentric ergometer exercise bout on the e-bike at $50 \%$ of their individual $P_{\max }$ for $15 \mathrm{~min}$ (see Fig. 1).

\section{Functional analysis}

The creatine kinase (CK) activity in the plasma was measured before the eccentric ergometer exercise and after $3,8,24,48$, and $96 \mathrm{~h}$ of recovery. The subjects also indicated their muscle soreness on a $0-10$ visual analogue scale (VAS) [5] at 3, 8, 24, 48, 72, and $96 \mathrm{~h}$ postexercise.

Muscle strength was assessed by counter movement and squat jumps before and 1 and 4 days after the eccentric ergometer exercise. A one-dimensional force platform and associated software (Quattro Jump ${ }^{\circledR}$, Kistler, Switzerland) was used to analyze jump parameters.

\section{Muscle sampling}

Before the eccentric exercise bout and at 1, 3, 8, and $24 \mathrm{~h}$ postexercise, fine-needle biopsies (14 gauge, single-use needles; Medilink, Pressagona, Switzerland) were taken from the vastus lateralis muscle [21] (Fig. 1). Our previous study of similar design but with concentric exercise [37] suggested that a gene transcript response to a single bout of
Fig. 1 Experimental protocol timeline. Muscle biopsies are taken before and after a single bout of mild eccentric ergometer exercise. Delayed onset of muscle soreness $(D O M S)$ peaks between 24 and $48 \mathrm{~h}$ of recovery from an eccentric single bout

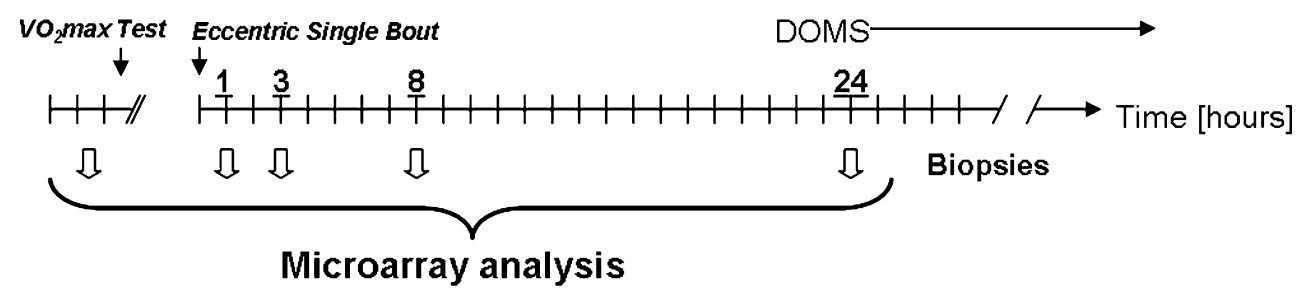


exercise is completed within $24 \mathrm{~h}$. We therefore stopped sampling muscle tissue after $24 \mathrm{~h}$. Muscle samples were immediately frozen in isopentane cooled by liquid nitrogen and stored in liquid nitrogen until analysis.

\section{Microarray analysis}

Total RNA was isolated from $25 \mu \mathrm{m}$ cryosections of the muscle biopsies as described previously [41]. The amount of isolated RNA was determined with the Ribo Green assay (Juro Supply, Lucerne, Switzerland). Subsequently, microarray experiments were carried out using custom-designed low-density Atlas complementary deoxyribonucleic acid (cDNA) expression arrays (BD Biosciences, Allschwil, Switzerland) as described [9]. The array held 231 double-spotted probes of human cDNAs associated with particular aspects of skeletal muscle function. Additionally, cDNA probes for an internal reference, $18 \mathrm{~S}$ ribosomal RNA (rRNA), were included on the array. Batches of five samples (all five time points of one individual) were processed simultaneously. ${ }^{32} \mathrm{P}$ Deoxyadenosine triphosphate (dATP)-labeled cDNA was generated from $1.2 \mu \mathrm{g}$ of total RNA by using the 231 gene-specific primers supplied. Probe synthesis for the measurement of the internal 18S rRNA reference was carried out in parallel. Total RNA $(0.3 \mu \mathrm{g})$ of each sample was, respectively, run for the generation of ${ }^{32} \mathrm{P}$ dATP-labeled cDNA with a specific primer for $18 \mathrm{~S}$ rRNA. Arrays were hybridized with a mix of total cDNA and $18 \mathrm{~S}$ cDNA diluted 1:1800. After 7 days of exposure, a phosphor imager (Molecular Dynamics, Sunnyvale, CA) was used to detect signal intensities.

Array evaluation

A template was created using the AIDA Array Metrix software (Raytest Schweiz AG, Urdorf, Switzerland). The raw signals, given as the sum of pixel intensities, were determined from the average signal intensities of the two corresponding dots. This mode calculates the average pixel intensity in a ring around each spot. The background intensity was estimated from 54 dots on each array. Transcripts were considered "detected" when the corresponding signal intensity was $30 \%$ above background in at least four of the six possible filter hybridizations for one time point. Microarray and detection procedures were identical to those used by Schmutz et al. [37].

Statistical analysis

Raw data were background-corrected and logarithmized to the base of 2. Standardization was performed by subtracting the 18S rRNA from the value of each transcript. Standardization to $18 \mathrm{~S}$ rRNA values was chosen because rRNA represents a major portion of the total RNA (i.e. 27\%) [12, 24].
To determine whether a signal of a particular messenger RNA (mRNA) was significantly $(p \leq 0.05)$ different or showed a tendency $(0.05<p \leq 0.1)$ throughout the time course, each detected gene was tested with the Friedman analysis of variance (ANOVA; Statistica 6.1; StatSoft [Europe], Hamburg, Germany). The nonparametric Friedman ANOVA was used to account for the paired design of the sampling. To identify significant differences between before and after the single exercise bout, the paired Wilcoxon test was applied $(p \leq 0.05)$. No adjustments were made for multiple testing. The results were validated with the L2 permutation regression analysis whereby expressional changes were identified as outliers to a linear regression line in scatter plots. Raw values of all detected gene transcripts were included in this analysis. This approach is justified by the robust-linear relationship between RNA expression levels from different individuals [14]. While the Friedman ANOVA is based on a paired comparison of the value of each time point to the prebiopsy value, the L2 regression is a compound analysis of all time points in a single regression.

The physiological variables of this study (CK activity, evaluation of muscle soreness [VAS] and jump height in the squat and counter movement jump [CMJ]) were analyzed using a nonparametric ANOVA with a Wilcoxon post-hoc test. Significance was accepted for $p \leq 0.05$ for all variables.

\section{Results}

Physiology

Subjects were of age $22 \pm 2.6$ years (mean \pm SD), height $176.8 \pm 6.7 \mathrm{~cm}$, weight $69.9 \pm 12.3 \mathrm{~kg}$, lean body mass $61.5 \pm$ $7.4 \mathrm{~kg}$, BMI $22.3 \pm 3.0 \mathrm{~kg} / \mathrm{m}^{2}, \mathrm{VO}_{2 \max } 45.1 \pm 6.0 \mathrm{ml} \mathrm{min}{ }^{-1}$ $\mathrm{kg}^{-1}$, and $P_{\max } 260 \pm 31 \mathrm{~W} / \mathrm{kg}$. After the eccentric ergometer exercise bout, all six individuals showed a significant reduction in the jumping height of the CMJ (-6.4\%) $24 \mathrm{~h}$ postexercise but recovered to normal levels after $96 \mathrm{~h}$ $(+8.8 \%$ higher than value after $24 \mathrm{~h})$. The decrease in squat jump height did not reach the level of significance $24 \mathrm{~h}$ after the eccentric exercise bout $(-2.4 \%)$ but was significantly higher after $96 \mathrm{~h}$ compared to the value after $24 \mathrm{~h}$ $(+5.4 \%)$, indicating a similar trend as seen for the CMJ (Table 1).

CK activity was significantly increased until day 2 after the eccentric exercise (see Table 1). However, CK values were not altered to the same extent as observed after more intense eccentric exercise [8]. A VAS for muscle pain showed a strong induction of delayed onset of muscle soreness (DOMS) that peaked between 24 and $48 \mathrm{~h}$ and stayed elevated until day 4 (see Table 1). 
Table 1 Physiological results

\begin{tabular}{|c|c|c|c|c|c|c|c|}
\hline Time point (h) & 0 & 3 & 8 & 24 & 48 & 72 & 96 \\
\hline SJ (height in $\mathrm{cm}$ ) & $41 \pm 5.9$ & & & $40.5 \pm 4.4$ & & & $42.7 \pm 5.1^{\mathrm{a}}$ \\
\hline $\mathrm{SJ}\left(P_{\max }\right.$ in $\left.\mathrm{W} / \mathrm{kg}\right)$ & $50.2 \pm 3.2$ & & & $47.4 \pm 2.9$ & & & $51.0 \pm 3.1^{\mathrm{a}}$ \\
\hline CMJ (height in $\mathrm{cm}$ ) & $43.5 \pm 3.6$ & & & $40.7 \pm 3.1^{\mathrm{a}}$ & & & $44.3 \pm 1.7^{\mathrm{a}}$ \\
\hline $\mathrm{CMJ}\left(P_{\max }\right.$ in $\left.\mathrm{W} / \mathrm{kg}\right)$ & $48.6 \pm 2.8$ & & & $46.7 \pm 2.4^{\mathrm{a}}$ & & & $49.7 \pm 2.3^{\mathrm{a}}$ \\
\hline CK in blood (U/I) & $91.5 \pm 26.5$ & $122.1 \pm 21.8^{\mathrm{a}}$ & $150.6 \pm 47.7^{\mathrm{a}}$ & $152.0 \pm 58.1$ & $128.8 \pm 34.4^{\mathrm{a}}$ & & $82.4 \pm 14.2$ \\
\hline VAS $(0,10)$ & $0.3 \pm 0.2$ & $1.5 \pm 1.0^{\mathrm{a}}$ & $2.7 \pm 2.9^{\mathrm{a}}$ & $5.9 \pm 1.4$ & $5.6 \pm 4.2$ & $3.0 \pm 1.2$ & $1.5 \pm 1.1$ \\
\hline
\end{tabular}

Mean of height, $P_{\max }$ of squat jump (SJ), and counter movement jump (CMJ) \pm standard error are given. Characteristics of DOMS: Creatine kinase (CK) activity and visual analogue scale (VAS). Significantly changed values from one time point to the other are marked with a superscripted "a"

\section{Microarray analysis}

Microarray analysis of 231 gene transcripts yielded a total of 147 detected gene transcripts. The time course of these, when analyzed together, showed a significant downregulation (Fig. 2). From the 147 detected gene transcripts, 80 turned out to have significantly changed throughout the time course (Friedman ANOVA). Out of these 80 transcripts, 58 were significantly changed at least at one time point throughout recovery (Wilcoxon test). Only seven of these were upregulated, while 51 were downregulated (Table 2). The downregulation of most of these transcripts was apparent already after $1 \mathrm{~h}$ postexercise and lasted over the entire observation period of $24 \mathrm{~h}$. Expression levels of many early downregulated mRNAs were significant higher at 24 than $1 \mathrm{~h}$ postexercise (see Table 2). This is indicative for the recovery of the overall gene transcript levels (Fig. 2). A few regulatory factors were upregulated. Among these, we found an early induction of mRNAs for c-jun and ubiquitin $\mathrm{C}$ (UBC) $1 \mathrm{~h}$ post exercise. Later, between 3 and $24 \mathrm{~h}$ postexercise,

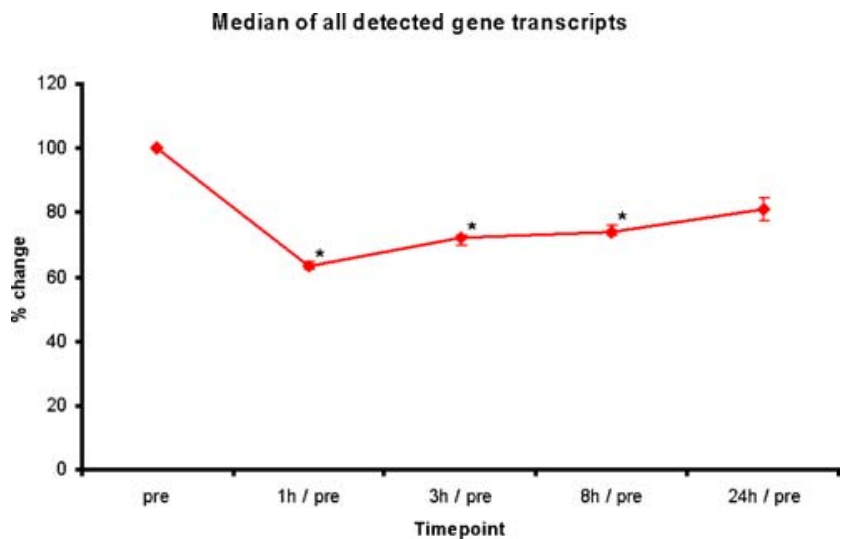

Fig. 2 The overall gene response. Median values and 95\% confidence intervals of all detected gene transcripts, relative ratio (in percent) of the different time points $(1,3,8$, and $24 \mathrm{~h}$ postexercise) to the prebiopsy. Friedman ANOVA shows a significant effect of time $(p=$ $0.013)$, Asterisk, significant downregulation compared to previous value $(p<0.05)$
mRNA levels for myogenic factor 6 (MYF6), cyclindependent kinase inhibitor $1 \mathrm{~A}$ (p21), tubulin alpha 1 (TUBA1), insulin-like growth factor binding protein 4 (IGFBP4), and interleukin 6 receptor (IL6R) were enhanced. All changes observed in transcript levels were validated, using the permutation-based L2 regression analysis (see supplemental Table 2). A close match between the two statistical analyses was found.

\section{Discussion}

\section{Study limitations}

An important limitation of this study is the relatively low number of subjects $(n=6)$ that could be included. It therefore seemed justified to use a relatively "coarsegrained" statistical approach (the Friedman ANOVA) to describe the dominant effects of the eccentric training intervention. This was done in particular with regard to the companion study on concentric exercise where the same number of subjects performed a concentric exercise and biopsies were analyzed with the identical molecular and statistical procedures. To have a more detailed view of potential changes related to our experimental intervention, we carried out a more sensitive analysis (L2 regression analysis with corrections for false discover rate) in addition to the Friedman ANOVA [12]. We have previously used this technique to identify muscle transcript level changes in a microarray study of a rat soleus muscle subjected to hindlimb suspension and subsequent reloading [14, 41]. The L2 regression analysis detects 90 transcripts that are significantly altered throughout the time course. This is a higher number than with the nonparametric Friedman ANOVA (58 transcripts). It shows a broadly similar signature of transcript level responses as the Friedman ANOVA but with a potentially higher resolving power. We find that the L2 regression analysis confirms and extends the Friedman ANOVA results and may allow for a finer grain view of the transcriptional events. 
Table 2 Effect of an eccentric single bout on the gene transcript signature

Median values of $18 \mathrm{~S}$ standardized transcript levels relative to the prebiopsy and the 1-h biopsy, respectively, are indicated. When the field is blank, the gene is not detected. Normal gene name: significant changes throughout the time course; Italic gene name: tendency in the time course. Significant downregulated gene transcripts $(p \leq 0.05)$ are indicated in gray and significant upregulated gene transcripts in black

\begin{tabular}{|c|c|c|c|c|c|c|c|c|c|}
\hline & Category & Gene & GonBank ID & p F-Anova & 1h/pre & $3 \mathrm{~h} / \mathrm{pre}$ & 8h/pre & $24 \mathrm{~h} /$ pre & $24 \mathrm{~h} / 1 \mathrm{~h}$ \\
\hline \multirow{11}{*}{ 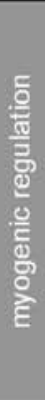 } & \multirow{4}{*}{ cell cycle } & MEF 2B & $x 63380$ & 0.015 & 0.75 & 0.44 & 0.62 & 0.66 & 0.88 \\
\hline & & MYF4 & $\times 17651$ & 0.026 & 0.52 & 0.85 & 1.10 & 1.61 & 3.11 \\
\hline & & MYF 6 & $\times 52011$ & 0.078 & 1.18 & 2.18 & 1.60 & 1.81 & 1.54 \\
\hline & & $S R F$ & 03181 & 0056 & 0.64 & 0.55 & 0.54 & 0.53 & 0.82 \\
\hline & proliferation & CD34 & M81 104 & 0.002 & 0.36 & & & 0.55 & 1.54 \\
\hline & \multirow{6}{*}{ hormonal } & IGFBP4 & M62403 & 0.020 & & & & 2.90 & \\
\hline & & IGFBP5 & M65062 & 0.003 & 0.63 & 0.92 & 0.56 & 0.47 & 0.75 \\
\hline & & IGF2 & M29645 & 0.005 & 0.67 & 1.03 & 0.80 & 0.60 & 0.89 \\
\hline & & ILER & M20568 & 0.097 & & & 3.09 & & \\
\hline & & LGALS1 & .04456 & 0.07 & 0.64 & 0.73 & 0.68 & 0.62 & 0.97 \\
\hline & & p21 & L.256 10 & 0.006 & 0.51 & 0.91 & 2.99 & 0.97 & 1.92 \\
\hline \multirow{5}{*}{ 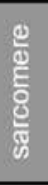 } & \multirow{5}{*}{ myofiber } & MYH2MYHC IIA & AF111784 & 0.005 & 0.51 & 0.74 & 0.61 & 0.56 & 1.10 \\
\hline & & MYHAMMYHC & AF111183 & ODEB & 0.59 & 0.66 & 0.74 & 0.81 & 1.37 \\
\hline & & МYH7МуНСь & M58013 & 001 & 0.62 & 0.94 & 0.69 & 0.49 & 0.80 \\
\hline & & DES & U5816? & 0.005 & 0.51 & 0.74 & 0.70 & 0.60 & 1.17 \\
\hline & & Titin & X69490 & 0.029 & 0.57 & 0.98 & 0.71 & 0.99 & 1.73 \\
\hline \multirow{13}{*}{ 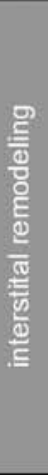 } & \multirow{2}{*}{ capillary } & ADORA1 & $\$ 56143$ & 0.024 & 0.82 & 0.71 & 1.10 & 1.37 & 1.67 \\
\hline & & ANG & M11567 & 0.048 & 0.34 & & & 0.42 & 1.22 \\
\hline & \multirow{2}{*}{ cytoskeleton } & CCT1 & $\times 52882$ & 0.036 & 0.91 & 0.54 & 0.83 & 1.24 & 1.36 \\
\hline & & TUBA1 & Koos5s & 0004 & 0.68 & 0.72 & 1.03 & 1.41 & 2.08 \\
\hline & \multirow{6}{*}{ degradation } & MMP 8 & 05556 & 0.082 & 0.69 & 0.38 & 0.51 & 0.58 & 0.85 \\
\hline & & MMP11 & X57766 & 0.053 & 0.62 & 0.57 & 0.78 & 1.24 & 2.00 \\
\hline & & MMP14 & D26512 & 0.002 & 0.56 & 0.45 & 0.40 & 0.49 & 0.87 \\
\hline & & MMP15 & 248482 & 0.032 & 0.69 & 0.40 & 0.63 & 0.68 & 0.98 \\
\hline & & PLAT & M15513 & 0.014 & 0.69 & 0.51 & 0.62 & 0.68 & 0.99 \\
\hline & & UBC & M26880 & 0.048 & 2.04 & 1.25 & 2.15 & 3.22 & 1.58 \\
\hline & \multirow{3}{*}{ ECM } & COL1A1 & Ko1228 & 0.023 & 0.34 & 0.33 & 0.37 & 0.49 & 1.43 \\
\hline & & TNC & $x 78565$ & 0.008 & 0.56 & 0.56 & 1.00 & 1.47 & 2.66 \\
\hline & & WVF & M10321 & 0.038 & 0.63 & 0.64 & 0.61 & 0.88 & 1.40 \\
\hline \multirow{13}{*}{ 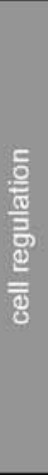 } & \multirow{5}{*}{ proliferation } & ADMR & BC034761 & 001 & 0.38 & 0.16 & 0.17 & 0.21 & 0.57 \\
\hline & & FGFR1 & M37722 & 0.012 & 0.47 & 0.40 & 0.74 & 1.20 & 2.54 \\
\hline & & FGFR4 & $\llcorner 03840$ & 0.032 & 0.34 & 0.29 & 0.28 & 0.29 & 0.85 \\
\hline & & ITGB1 & X07979 & 0.027 & 0.35 & 0.21 & 0.40 & 0.41 & 1.16 \\
\hline & & LAMR1 & U43901 & 0.045 & 0.84 & 0.98 & 0.97 & 1.53 & 1.82 \\
\hline & \multirow{4}{*}{ transcription } & HIF $1 \mathrm{~b}$ & M69233 & 0002 & 0.56 & 0.43 & 0.54 & 0.67 & 1.19 \\
\hline & & EPAS1 & U81984 & 0.066 & 0.48 & 0.57 & 0.65 & 0.73 & 1.53 \\
\hline & & PPARA & L02932 & 0.004 & 0.80 & 0.51 & 0.47 & 0.79 & 0.99 \\
\hline & & PPARG & $\angle 40904$ & 0.097 & 0.87 & 0.66 & 0.72 & 1.29 & 1.48 \\
\hline & \multirow{4}{*}{ signaling } & c.jun & 04111 & 0.003 & 2.85 & 0.74 & 0.66 & 0.68 & 0.24 \\
\hline & & DMPK & L19268 & 0003 & 0.45 & & & 1.17 & 2.60 \\
\hline & & ITGAB & L36531 & 0.019 & 0.79 & 0.72 & 0.57 & 1.15 & 1.47 \\
\hline & & VCAMI & X53051 & 0063 & 0.73 & 0.48 & 0.84 & 0.76 & 1.04 \\
\hline \multirow{16}{*}{ 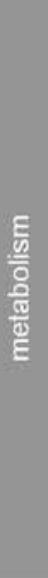 } & & ACADVL & $D 43682$ & 0.019 & 0.61 & 0.69 & 0.70 & 0.66 & 1.07 \\
\hline & & CPTI & D87812 & 0.004 & 0.54 & 0.62 & 0.46 & 0.41 & 0.76 \\
\hline & beth oridion & $\mathrm{DCl}$ & L24774 & 0.011 & 0.58 & 0.67 & 0.55 & 0.52 & 0.90 \\
\hline & 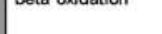 & $E C H 1$ & บ16660 & 0.097 & 0.72 & 0.64 & 0.56 & 0.51 & 0.70 \\
\hline & & ECHS1 & D13900 & 0038 & & & & 0.33 & \\
\hline & & HADHB & D16481 & 0.07 & 0.53 & 0.52 & 0.59 & 0.89 & 1.67 \\
\hline & HOmptom & GLUT4 & M20747 & 0.006 & 0.50 & & & & \\
\hline & 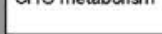 & SCP2 & M75883 & 0.016 & 0.77 & 0.61 & & 0.73 & 0.96 \\
\hline & detoxification & GPX5 & $A .005277$ & 0.078 & 0.78 & 0.68 & 0.56 & 0.64 & 0.82 \\
\hline & gycolysis & ALDOA & M11560 & 0.021 & 0.74 & 0.96 & 0.60 & 0.58 & 0.79 \\
\hline & $\mathrm{O}_{2}$ storage & MB & M14603 & 0.028 & 0.90 & 0.94 & 0.77 & 0.78 & 0.87 \\
\hline & andr & SOD1 & M13267 & 0.074 & 0.82 & 0.74 & 0.74 & 1.03 & 1.26 \\
\hline & readox & SOD3 & 02947 & 0.009 & 0.67 & 0.62 & 0.64 & 0.81 & 1.21 \\
\hline & & $\mathrm{CA} 3$ & M29458 & 0.015 & 0.86 & 1.27 & 0.70 & 0.66 & 1.23 \\
\hline & respiration & $\operatorname{cox} 5 B$ & M19961 & 0082 & 1.10 & 1.17 & 0.94 & 0.90 & 0.82 \\
\hline & & UCP3 & AF011449 & 0.003 & 0.71 & 0.68 & 0.55 & 0.65 & 0.91 \\
\hline
\end{tabular}


mRNA decline after mild eccentric exercise

The prominent finding of this study is a gene response characterized by a general decline of muscle-specific mRNA concentrations right after the exercise, followed by a recovery of transcript levels close to control values within $24 \mathrm{~h}$.

There are two basic mechanisms that can influence mRNA levels: transcription, which is generally under positive control, and mRNA degradation, which is related to prior translation of the mRNAs [35]. The fast and substantial suppression of most mRNA levels could thus be explained by a lack of de novo synthesis of mRNAs and by an increased degradation (see also discussion of UBC regulation below). An early decline in mRNA concentrations at $1 \mathrm{~h}$ has been observed before [37], but mRNA concentrations of metabolic factors were subsequently increased $8 \mathrm{~h}$ postexercise. The observed low mRNA concentrations at $8 \mathrm{~h}$ in the present study seem to be a consequence of a different time course of transcriptionrelated accretion and translation-related degradation of mRNA. Both concentric and eccentric resistance exercises lead to an increase in muscle protein synthesis $3 \mathrm{~h}$ after exercise bouts [30]. There is evidence suggesting a protection of mitochondrial RNAs from degradation after high-repetitive low-load contractions [42]. In the eccentric experiment, mRNA concentrations remained low at a time $(8 \mathrm{~h})$, when there was an expected enhancement of mRNA transcription [7].

\section{Upregulated gene transcripts}

The upregulation of a few gene transcripts points to the activation of distinct biological processes are as follows.

Myogenesis Several of the observed cell-regulatory RNAs relate to myogenesis. The immediate upregulation of c-jun and UBC represents its de novo RNA synthesis, which was shown to occur in many cell types after stress [22]. Upregulation of c-jun induces a partial activation of the cell cycle (G1) via the c-jun $\mathrm{NH}_{2}$-terminal kinase pathway. This finding relates to the transient elevation of the myogenic factor MYF6 (also called MRF4) mRNA after $3 \mathrm{~h}$ (see Table 2) and the temporary increase in this mRNA 2-4 $\mathrm{h}$ after single-resistance exercise [43]. The transcripts for other myogenic master regulators, MYOD1 and myogenin, were not altered in our study (Table 2 and supplemental Table 2). We therefore view this signature of response as a partial activation of the myogenic pathways. The upregulation of IGFBP4 and downregulation of IGFBP5 expression reproduces the effect of muscle loading in rodents $[1,9]$. This conserved response indicates that the muscle hypertrophy-associated IGF-1 system [3, 32] is subjected to a complex regulation in higher vertebrates.
This finding coincides with the upregulation of the cyclindependent kinase inhibitor $\mathrm{p} 21$. Changes in the p21 mRNA after resistance exercise are coregulated with IGFBP4 mRNA changes and related to cytoskeletal remodeling with muscle differentiation, which is indicated by the upregulation of TUBA1 mRNA $24 \mathrm{~h}$ after the eccentric exercise $[4,39]$. Our observation of an early rise in UBC mRNA is compatible with other studies, which showed an increase in UBC mRNA and protein levels after eccentric exercise [40]. These authors suggested that these changes were responsible for the observed increase in muscle proteolysis, as this was also indicated in another eccentric study with high exercise intensity [11]. The more than twofold upregulation of UBC mRNA in the current study points to enhanced tagging of proteins for proteolytic degradation by the proteolytic ubiquitin pathway. It is possible that enhanced protein degradation could also include degradation of muscle-specific mRNAs and explain our results $[6,16]$.

Damage The low increase in CK activity points to a moderate damage response to the eccentric ergometer exercise. This may suggest a low level of cell infiltration. We do, however, not observe a mRNA response of interleukin (IL) 6 and Tenascin-C, both markers of muscle damage $[14,29]$. This relates to the grading of the inflammation response with respect to muscle damage and elevated CK values after mild and hard eccentric protocols [8]. Instead, we see an upregulation of the mRNA for the IL6R. This puzzling observation implies that altered expression of IL6 receptor has to be considered for interpretations on damage-induced IL6 signaling after eccentric contractions [23].

\section{Gene signature comparisons}

In a previous study, we investigated the gene expression signature after concentric exercise with an identical microarray technology and statistical analysis (Friedmann ANOVA) [37]. Although this concentric exercise was performed at different duration and intensity, it allows us to compare these exercises with minimal uncertainties introduced by the technical procedures. Moreover, the initial load of the two training sets, when applied and adapted over a longer time period, would result in a specific and measurable training response [25, 37]. This comparison is interesting because eccentric contractions require less motor unit activation and consume less oxygen and energy for a given muscle force than concentric contractions [27]. They therefore represent training modalities with a different application profile. Schmutz et al. [37] showed that concentric exercise induces an upregulation of several metabolic pathways including glycolysis, beta-oxidation, respiration, the Krebs cycle, and detoxification, after an 
initial $(1 \mathrm{~h})$ decline in mRNA levels [37]. This upregulation was not observed in the current study (see supplemental Table 2). It thus seems that the metabolic stimulus of the eccentric exercise was not sufficient to induce upregulation of these pathways involved in aerobic energy generation and mitochondrial biogenesis. This finding is consistent with our findings showing mRNA for cytochrome c oxidase subunit IV to be significantly downregulated after eccentric and upregulated after concentric type of exercise in stable coronary artery disease patients [44]. We only found evidence for a slight mitochondrial reaction. The L2 regression analysis detects weakly upregulated mRNA levels of cytochrome c oxidase subunit $5 \mathrm{~b}$, cytochrome $\mathrm{c}$ oxidase 1 , and cytochrome $\mathrm{c}$.

Our observations also contrast with the findings from Chen et al. [7], who investigated the gene response to maximal low-repetitive eccentric exercise in humans with an Affymetrix Human Genome microarray. This investigation found no consistent downregulation of any gene transcript but showed a limited increase in expression of gene transcripts involved in the inflammatory response, e.g., IL1 receptor and Tenascin-C. Eccentric exercise is also associated with exercise-induced muscle damage, proteolysis [11], and increased serum levels of IL6 [8, 29] and CK [8]. This supports the idea that maximal eccentric exercise leads to mechanical damage of myofibers and the stimulation of an inflammatory response in a load-dependent manner. Because we did not observe markers of muscle damage, we suggest that the mechanical load was not high enough to activate a more pronounced inflammatory response.

\section{Events underlying muscle hypertrophy}

The molecular observations in the eccentrically challenged vastus lateralis muscle are astonishing with regard to the observed hypertrophy response to the same type of exercise, when carried out repetitively. It was shown that 8 weeks of mild eccentric exercise lead to an increase in capillary-to-fiber ratio of $47 \%$ and fiber cross-sectional area of $52 \%$ [25].

Based on the results of the Friedman ANOVA, we have to revise the hypothesis of a general upregulation of transcripts relevant to muscle growth. The early selective upregulation of c-jun and MYF6 suggests a mechanodependent activation of some aspects of myogenesis. A load-dependent activation of jun pathway in situ [26] and c-jun expression in myonuclei and interstitium has been seen before with running exercise with a high component of eccentric loading [34]. This notion of an activated myogenesis was also corroborated by the results of the L2 regression analysis, which identified enhanced amount of myogenic factor MYF4 and desmin mRNA. High-load single bout of eccentric exercise was shown to increase
mRNA levels of the myogenic factors MYF6, MYOD, and myogenin in humans [43]. This statistical test indicating enhanced message for the ribosomal proteins (RPS9 and RPS29) and 18S and 28S ribosomal RNAs after 8 and $24 \mathrm{~h}$ (see supplemental Table 2) also provided circumstantial evidence for activation of protein translation. This finding points to the key role of muscle loading as a trigger for protein synthesis [19] and the suggested enhanced ribosome number after a 2-month period of eccentric training [15]. The latter observations may be indicative of an elevated translation capacity after eccentric exercise and thus eventually explain part of the observed decline in mRNA levels. In support of this, it was found that eccentric contractions are more effective than concentric contractions in stimulating protein synthesis $[2,10]$. Thus, we conclude that there is some molecular evidence for a transcriptional basis of the elevated protein synthesis with eccentric ergometer exercise, which critically depends on the applied mechanical load.

\section{Conclusion}

In this study, we investigated the temporal response of muscle gene expression to a single bout of mild eccentric ergometer exercise. We had to revise our hypothesis that we could identify a major upregulation of transcripts relevant for processes supporting muscle growth within the first $24 \mathrm{~h}$ of recovery. Instead, we found that the mRNAs of almost all important muscle regulatory gene transcripts are significantly downregulated and take close to (or more than) $24 \mathrm{~h}$ to revert to pre-exercise values. This study demonstrates for the first time that mild eccentric exercise has a molecular signature distinctly different from intensive concentric exercise as well as from maximal eccentric exercise.

Acknowledgments The support of the Eidgenössische Sport Kommission (ESK) and the University of Bern is gratefully acknowledged. Special thanks are addressed to Christoph Lehmann for technical support, Felix Weinstein for assistance in the L2 regression analysis, and Dunja Minder for editorial proof-reading of the manuscript.

\section{References}

1. Awede B, Thissen J, Gailly P, Lebacq J (1999) Regulation of IGF-I, IGFBP-4 and IGFBP-5 gene expression by loading in mouse skeletal muscle. FEBS Lett 461:263-267

2. Baar K, Esser K (1999) Phosphorylation of p70(S6k) correlates with increased skeletal muscle mass following resistance exercise. Am J Physiol 276:C120-C127

3. Bamman MM, Shipp JR, Jiang J, Gower BA, Hunter GR, Goodman A, McLafferty CL Jr, Urban RJ (2001) Mechanical load increases muscle IGF-I and androgen receptor mRNA concentrations in humans. Am J Physiol Endocrinol Metab 280: E383-E390 
4. Bickel CS, Slade J, Mahoney E, Haddad F, Dudley GA, Adams GR (2005) Time course of molecular responses of human skeletal muscle to acute bouts of resistance exercise. J Appl Physiol 98:482-488

5. Carlsson AM (1983) Assessment of chronic pain. I. Aspects of the reliability and validity of the visual analogue scale. Pain 16:87-101

6. Cascone PJ, Schwartz LM (2001) Post-transcriptional regulation of gene expression during the programmed death of insect skeletal muscle. Dev Genes Evol 211:397-405

7. Chen YW, Hubal MJ, Hoffman EP, Thompson PD, Clarkson PM (2003) Molecular responses of human muscle to eccentric exercise. J Appl Physiol 95:2485-2494

8. Clarkson PM, Hubal MJ (2002) Exercise-induced muscle damage in humans. Am J Phys Med Rehabil 81:S52-S69

9. Dapp C, Schmutz S, Hoppeler H, Fluck M (2004) Transcriptional reprogramming and ultrastructure during atrophy and recovery of mouse soleus muscle. Physiol Genomics 20:97-107

10. Eliasson J, Elfegoun T, Nilsson J, Kohnke R, Ekblom B, Blomstrand E (2006) Maximal lengthening contractions increase p70 S6 kinase phosphorylation in human skeletal muscle in the absence of nutritional supply. Am J Physiol Endocrinol Metab 291:E1197-E1205

11. Feasson L, Stockholm D, Freyssenet D, Richard I, Duguez S, Beckmann JS, Denis C (2002) Molecular adaptations of neuromuscular disease-associated proteins in response to eccentric exercise in human skeletal muscle. J Physiol 543:297-306

12. Fluck M, Dapp C, Schmutz S, Wit E, Hoppeler H (2005) Transcriptional profiling of tissue plasticity: role of shifts in gene expression and technical limitations. J Appl Physiol 99:397-413

13. Fluck M, Hoppeler H (2003) Molecular basis of skeletal muscle plasticity - from gene to form and function. Rev Physiol Biochem Pharmacol 146:159-216

14. Fluck M, Schmutz S, Wittwer M, Hoppeler H, Desplanches D (2005) Transcriptional reprogramming during reloading of atrophied rat soleus muscle. Am J Physiol Regul Integr Comp Physiol 289:R4-R14

15. Friden J (1984) Changes in human skeletal muscle induced by long-term eccentric exercise. Cell Tissue Res 236:365-372

16. Gardrat F, Montel V, Raymond J, Azanza JL (1999) Degradation of an ubiquitin-conjugated protein is associated with myoblast differentiation in primary cell culture. Biochem Mol Biol Int 47:387-396

17. Higbie EJ, Cureton KJ, Warren GL III, Prior BM (1996) Effects of concentric and eccentric training on muscle strength, cross-sectional area, and neural activation. J Appl Physiol 81:2173-2181

18. Hood DA, Irrcher I, Ljubicic V, Joseph AM (2006) Coordination of metabolic plasticity in skeletal muscle. J Exp Biol 209:2265-2275

19. Hornberger TA, Stuppard R, Conley KE, Fedele MJ, Fiorotto ML, Chin ER, Esser KA (2004) Mechanical stimuli regulate rapamycinsensitive signalling by a phosphoinositide 3-kinase-, protein kinase B-and growth factor-independent mechanism. Biochem J 380:795-804

20. Hortobagyi T, Hill JP, Houmard JA, Fraser DD, Lambert NJ, Israel RG (1996) Adaptive responses to muscle lengthening and shortening in humans. J Appl Physiol 80:765-772

21. Howald H, Hoppeler H, Claassen H, Mathieu O, Straub R (1985) Influences of endurance training on the ultrastructural composition of the different muscle fiber types in humans. Pflugers Arch 403:369-376

22. Hunter T, Karin M (1992) The regulation of transcription by phosphorylation. Cell 70:375-387

23. Keller P, Penkowa M, Keller C, Steensberg A, Fischer CP, Giralt M, Hidalgo J, Pedersen BK (2005) Interleukin-6 receptor expression in contracting human skeletal muscle: regulating role of IL-6. FASEB J 19:1181-1183
24. Larson DE, Zahradka P, Sells BH (1991) Control points in eucaryotic ribosome biogenesis. Biochem Cell Biol 69:5-22

25. Lastayo PC, Pierotti DJ, Pifer J, Hoppeler H, Lindstedt SL (2000) Eccentric ergometry: increases in locomotor muscle size and strength at low training intensities. Am J Physiol Regul Integr Comp Physiol 278:R1282-R1288

26. Martineau LC, Gardiner PF (2001) Insight into skeletal muscle mechanotransduction: MAPK activation is quantitatively related to tension. J Appl Physiol 91:693-702

27. Meyer K, Steiner R, Lastayo P, Lippuner K, Allemann Y, Eberli F, Schmid J, Saner H, Hoppeler H (2003) Eccentric exercise in coronary patients: central hemodynamic and metabolic responses. Med Sci Sports Exerc 35:1076-1082

28. Nader GA (2006) Concurrent strength and endurance training: from molecules to man. Med Sci Sports Exerc 38:1965-1970

29. Pedersen BK, Ostrowski K, Rohde T, Bruunsgaard H (1998) Nutrition, exercise and the immune system. Proc Nutr Soc 57:43-47

30. Phillips SM, Tipton KD, Aarsland A, Wolf SE, Wolfe RR (1997) Mixed muscle protein synthesis and breakdown after resistance exercise in humans. Am J Physiol 273:E99-E107

31. Pilegaard H, Ordway GA, Saltin B, Neufer PD (2000) Transcriptional regulation of gene expression in human skeletal muscle during recovery from exercise. Am J Physiol Endocrinol Metab 279:E806-E814

32. Psilander N, Damsgaard R, Pilegaard H (2003) Resistance exercise alters MRF and IGF-I mRNA content in human skeletal muscle. J Appl Physiol 95:1038-1044

33. Puntschart A, Claassen H, Jostarndt K, Hoppeler H, Billeter R (1995) mRNAs of enzymes involved in energy metabolism and mtDNA are increased in endurance trained athletes. Am J Physiol 269:C619-C625

34. Puntschart A, Wey E, Jostarndt K, Vogt M, Wittwer M, Widmer HR, Hoppeler H, Billeter R (1998) Expression of fos and jun genes in human skeletal muscle after exercise. Am J Physiol 43:C129-C137

35. Sachs AB (1993) Messenger RNA degradation in eukaryotes. Cell 74:413-421

36. Sale D, MacDougall D (1981) Specificity in strength training: a review for the coach and athlete. Can J Appl Sport Sci 6:87-92

37. Schmutz S, Dapp C, Wittwer M, Vogt M, Hoppeler H, Fluck M (2006) Endurance training modulates the muscular transcriptome response to acute exercise. Pflugers Arch 451:678-687

38. Steiner R, Meyer K, Lippuner K, Schmid JP, Saner H, Hoppeler H (2004) Eccentric endurance training in subjects with coronary artery disease: a novel exercise paradigm in cardiac rehabilitation? Eur J Appl Physiol 91:572-578

39. Tassin AM, Maro B, Bornens M (1985) Fate of microtubuleorganizing centers during myogenesis in vitro. J Cell Biol 100:35-46

40. Willoughby DS, Taylor M, Taylor L (2003) Glucocorticoid receptor and ubiquitin expression after repeated eccentric exercise. Med Sci Sports Exerc 35:2023-2031

41. Wittwer M, Fluck M, Hoppeler H, Muller S, Desplanches D, Billeter R (2002) Prolonged unloading of rat soleus muscle causes distinct adaptations of the gene profile. FASEB J 16:884-886

42. Yan Z, Salmons S, Dang YI, Hamilton MT, Booth FW (1996) Increased contractile activity decreases RNA-protein interaction in the 3'-UTR of cytochrome c mRNA. Am J Physiol 271:C1157C1166

43. Yang Y, Creer A, Jemiolo B, Trappe S (2005) Time course of myogenic and metabolic gene expression in response to acute exercise in human skeletal muscle. J Appl Physiol 98:1745-1752

44. Zoll J, Steiner R, Meyer K, Vogt M, Hoppeler H, Fluck M (2006) Gene expression in skeletal muscle of coronary artery disease patients after concentric and eccentric endurance training. Eur $\mathrm{J}$ Appl Physiol 96:413-422 